\title{
AVALIAÇÃO DA ATIVIDADE MITOCONDRIAL EM ESPERMATOZÓIDES PÓS-COLHEITA E PÓS- DESCONGELAÇÃO DE CAPRINOS DAS RAÇAS BOER E ALPINA NO OUTONO E PRIMAVERA
}

\author{
(Evaluation of mitochondrial activity in spermatozoids after collecting and \\ posthawing in Boer and Alpine goats in autumn and spring)
}

\author{
CAVALCANTE, T.V.'; ESPER, C.R. ${ }^{2}$; FERREIRA, J.L.'; DIAS, F.E.F.'; \\ AZEVEDO, H.C. ${ }^{3}$; CORDEIRO, M.F. ${ }^{2}$; SOUZA, J.A.T. ${ }^{4}$ \\ 'Medicina Veterinária e Zootecnia/Universidade Federal do Tocantins/Araguaína-TO; \\ ${ }^{2}$ Faculdade de Ciências Agrárias e Veterinárias/Unesp/Jaboticabal-SP; \\ ${ }^{3}$ CNPTC - Centro Nacional de Pesquisa Tabuleiros Costeiros, EMBRAPA, Aracaju-SE; \\ ${ }^{4}$ Universidade Federal do Piauí/ Centro de Ciências Agrárias/Teresina-PI.
}

\begin{abstract}
RESUMO - Os espermatozóides caprinos realizam a respiração mitocondrial, mantendo um balanço energético necessário para o transporte e as demais funções celulares. Um teste objetivo de avaliação quantitativa e qualitativa da atividade mitocondrial em espermatozóides caprinos foi aplicado no sêmen de nove reprodutores das raças Boer $(n=5)$ e Alpina $(n=4)$ colhidos e congelados em diluidor TRIS em duas estações. A colheita do sêmen foi realizada com auxílio da vagina artificial, totalizando 135 ejaculados e foram confeccionados esfregaços com sêmen pós-colheita e pós-descongelação. Para a determinação da atividade mitocondrial foi utilizado o procedimento citoquímico desenvolvido e validado por HRUDKA (1987). Esta técnica é baseada na oxidação da 3,3'-diaminobenzidine (DAB) pela enzima em reação em cadeia, onde o reagente é polimerizado e depositado na bainha mitocondrial ao longo da peça intermediária dos espermatozóides. Foi determinado um índice médio de atividade citoquímica (IAC) de $74,37 \%, 74,37 \%$ no outono, $77 \%$ e $77,75 \%$ na primavera. E $85,62 \%$ e 85,93 no pós-colheita, $66 \%$ e $67,25 \%$ pós-descongelação para as raças Boer e Alpina, respectivamente. As variáveis raças, pós-colheita e pós-descongelação, classes (IAC), e estação foram estudadas por meio de ANOVA. Não foram observadas diferenças estatísticas para raças, classes, bem como para as estações. Entretanto, efeito significativo entre as amostras pós-colheita e pós-descongelação $(\mathrm{P}<0,01)$. Conclui-se que as células espermáticas de caprinos colhidos em duas estações do ano, e das raças estudadas, apresentam índice de atividade citoquímica similares, o que evidencia semelhante metabolismo aeróbico dos espermatozóides.
\end{abstract}

Palavras-chaves: espermatozóides, caprinos, atividade mitocondrial.

ABSTRACT - Caprine spermatozoa undergo glycolisis and mitocondrial respiration to keep the necessary energy balance to their transportation and other cellular functions. An objective test to evaluate quantitatively and qualitatively mitochondrial activity in caprine spermatozoa was applied in the analysis of semen samples of 9 bucks from Boer $(n=5)$ and Alpine $(n=4)$ breeds. Samples were collected and frozen in TRIS medium in two different year seasons. Semen collection was performed using artificial vagina to obtain 135 ejaculates, which were used to post-collection and post-frozen extended in sildes. To determine mitochondrial activity, a citochemical procedure developed and validated for HRUDKA (1987) was used. The technique is based on the enzymatic 3,3'-diaminobenzidine (DAB) oxidation, where the reagent is polymerized and attached to the mitochondrial membrane throughout the spermatic intermediary piece. A medium level of citochemical activity (IAC) was determined as being $74,37 \%$, $74,37 \%$ during Autumn and $77 \%$ e $77,75 \%$ during Spring for Boer and Alpine breeds, respectively. For post-collection IAC levels were $85,62 \%$ and 85,93 and for post-frozen $66 \%$ and $67,25 \%$ for Boer and Alpine breeds, respectively. ANOVA test was used to evaluate breed, post-collection, post-frozen, IAC rates and year season parameters. Differences were not noticed for breed, IAC rates and year season. Nonetheless, there was a significant effect among samples for post-collection and post-frozen analysis $(\mathrm{P}<0,01)$. It was concluded that caprine spermatozoa collected in two different year seasons, in the two breeds, presented similar IAC levels, what makes evident that there is similar aerobic spermatic metabolism.

Key-words: espermatozoa, caprine, mitochondrial activity. 


\section{Introdução}

$\mathrm{Na}$ rotina de análise de sêmen para a predição da fertilidade, a concentração, motilidade e morfologias espermáticas, são avaliadas, na tentativa de se obter informações sobre o estado da espermatogênese e do potencial de fertilização do espermatozóide.

Porém, estes testes são limitados e não podem avaliar a real habilidade de fertilização. Análises da função espermática têm obtido importância nas últimas décadas, já que as técnicas convencionais não têm demonstrado estimar acuradamente e repetidamente a fertilidade da amostra de sêmen (CORREA et al., 1997). Deste modo, o desenvolvimento de técnicas que buscam a avaliação do status funcional das organelas espermáticas (acrossomo, mitocôndria), ou a integridade de muitos componentes celulares, permite um diferente acesso para o problema (GRAHAM, 2001).

Os espermatozóides são células metabolicamente ativas que realizam tanto a glicólise como a respiração mitocondrial, mantendo um adequado balanço energético necessário para o transporte e as demais funções celulares (BARBOSA e ESPER, 1997).

O comprimento da peça intermediária dos espermatozóides tem sido considerado indicador biológico, que pode ser usado como preditor de características importantes em bovinos (LUKEFAHR e HOHENBOKEN, 1981). A peça intermediária é composta de mitocôndrias circundando a região proximal do flagelo, sendo possível que um aumento no seu comprimento poderia estar associado a uma maior taxa de fosforilação oxidativa, e assim com maior atividade metabólica influenciando na motilidade ou vitalidade da célula, já que as mitocôndrias são transformadoras de energia para a célula (EDDY, 1988). A perda oxidativa do DNA mitocondrial e da estrutura da membrana podem ser um fator de grande importância para explicar a diminuição da fertilidade e motilidade do sêmen criopreservado (CUMMINS et al. 1994), já que as mitocôndrias da peça intermediária do espermatozóide produz energia para suportar a motilidade; alterações no potencial da membrana mitocondrial pode ser um bom indicador da diminuição desta função (PEÑA et al. 2003).

Considerando tais aspectos, diretamente relacionados com o transporte espermático, decorrentes da estrutura morfológica, funcional e bioquímica da peça intermediária dos espermatozóides, e a necessidade de identificar características do espermatozóide, que reflitam na sua habilidade fertilizadora, o objetivo deste trabalho foi avaliar a influência de duas estações distintas dos reprodutores das raças Boer e Alpina com a seguinte prova in vitro: Atividade Mitoncondrial.

\section{Materiais e Métodos}

Local do experimento e animais: Após exame clínico geral e andrológico, foram selecionados nove reprodutores (com idade entre dois e cinco anos) das raças Boer $(n=5)$ e Alpina $(n=4)$, considerados normais e mantidos no Setor de Pequenos Ruminantes do Departamento de Reprodução Animal e Radiologia da FMVZ/ Unesp, Campus de Botucatu.

O experimento foi conduzido nas estações, reprodutiva (outono) e não reprodutiva (primavera) para colheita e congelação do sêmen e prova in vitro. Os machos foram mantidos em regime de criação intensiva, sob luminosidade natural, em uma latitude de $22^{\circ} 53^{\prime}$ 'S e longitude $48^{\circ} 29^{\prime} \mathrm{W}$, recebendo 0,9 $\mathrm{KgMS} / c a b e c ̧ a / d i a$ de ração completa (concentrado + volumoso) e disponibilidade ad libitum de sal mineral e água.

Prova in vitro - Avaliação Mitocondrial: A atividade citoquímica foi determinada usando o método descrito por HRUDKA (1987). Amostras de sêmen in natura e após descongelação foram incubadas em meio contendo diaminobenzidine (DAB-Sigma, Chemical Co., St. Louis, MO, USA) dissolvido em fosfato de sódio $0,15 \mathrm{M}$, pH 7,2, a uma concentração de $1 \mathrm{mg} / \mathrm{mL}$ e manuseado na ausência de luz.

Uma alíquota de $10 \mu \mathrm{L}$ (in natura) e $250 \mu \mathrm{L}$ (pós-descongelação) de sêmen foi adicionada a $1 \mathrm{~mL}$ e $750 \mu \mathrm{L}$, respectivamente, do meio de incubação, homogeneizada em tubo de ensaio aberto, colocada em banho-maria a $37^{\circ} \mathrm{C}$, durante 60 minutos, na ausência de luz. Para 
Avaliação da atividade mitocondrial em espermatozóides pós-colheita e pós-descongelação de caprinos...

cada ensaio realizado, um controle negativo foi obtido da incubação de uma amostra de sêmen aquecida a $100^{\circ} \mathrm{C}$ por cinco minutos e adicionada ao meio de incubação nas mesmas proporções do teste.

Após incubação foram preparados dois esfregaços de cada suspensão, fixados em formaldeído $10 \%$ por 10 minutos, lavados e secos ao ar. As avaliações dos espermatozóides foram feitas em objetiva 100x, sob imersão em óleo, usando microscopia de contraste de fase (Nikon, modelo SKT, Japan). Foram classificados 200 espermatozóides (200 por duplicata) por ejaculado.

A avaliação da atividade mitocondrial da peça intermediária dos espermatozóides obedeceu escala de quatro classes propostas por HRUDKA (1987), onde:

- Classe 1: Quase todas as mitocôndrias são ativas, ou seja, contêm um produto de reação colorida dando à bainha mitocondrial a aparência de um cilindro compacto e proeminente.

- Classe 2: A bainha mitocondrial aparece fragmentada, isto é, consiste em segmentos ativos e inativos com predominância dos ativos.

- Classe 3: Espermatozóides com menos da metade da bainha mitocondrial ativa e com poucas mitocôndrias ativas dispersas.

- Classe 4: Espermatozóides completamente inativos.

Cada classe da escala foi multiplicada por um fator de correção, como segue: 1:1,0;2:0,5; $3: 0,25 ; 4: 00$. Desta forma calcula-se o índice de atividade citoquímica (IAC), multiplicandose o número de espermatozóides encontrados em cada uma das classes pelo seu escore relativo.

Os testes foram feitos em duplicata de todos ejaculados (sêmen fresco e congelado) de cada reprodutor em cada estação.

Realizou-se uma análise de variância seguida do teste de comparação múltipla de Tukey-Kramer para cada uma das variáveis estudadas. Todas as informações foram analisadas usando SAS System v.8.2 (SAS, 2003), usando-se os procedimentos GLM, FREQ e TTEST, e utilizou-se um nível de significância de 0,01 para todos os testes de hipóteses.

\section{Resultados e Discussão}

Os valores médios do número de espermatozóides e o índice de atividade citoquímica nas amostras pós-colheita e pósdescongelação, no outono e na primavera, distribuídos nas quatro classes de atividade, das raças Boer e Alpina, encontram-se nas TABELAS 1 e 2, e FIGURA 1.

TABELA 1 - MÉDIAS E DESVIOS PADRÕES ( \pm ) AJUSTADOS POR QUADRADOS MÍNIMOS PARA O NÚMERO DE ESPERMATOZÓIDES E ÍNDICE DE ATIVIDADE CITOQUÍMICA (IAC), POR CLASSES, DE ACORDO COM HRUDKA (1987), AVALIADAS NO SÊMEN CAPRINO, PÓS-COLHEITA E PÓS-DESCONGELAÇÃO, DAS RAÇAS BOER E ALPINA, BOTUCATU-SP, 2002.

\begin{tabular}{|c|c|c|c|c|}
\hline \multirow[t]{2}{*}{ Classes } & \multicolumn{2}{|c|}{$\begin{array}{l}\text { Pós-colheita } \\
\text { № Sptz (IAC) }\end{array}$} & \multicolumn{2}{|c|}{$\begin{array}{c}\text { Pós-descongelação } \\
\text { № Sptz (IAC) }\end{array}$} \\
\hline & $\operatorname{Boer}(\mathrm{n}=41)$ & Alpina $(n=44)$ & Boer $(n=38)$ & Alpina $(n=41)$ \\
\hline 1 & $169 \pm 4^{\mathrm{a}}(84,5)$ & $169 \pm 4^{a}(84,5)$ & $109 \pm 4^{b}(54,5)$ & $107 \pm 3^{b}(53,5)$ \\
\hline 2 & $3 \pm 1^{\mathrm{b}}(0,75)$ & $2 \pm 1^{\mathrm{b}}(0,5)$ & $37 \pm 2^{a}(9,25)$ & $48 \pm 1^{a}(12)$ \\
\hline 3 & $3 \pm 1^{b}(0,37)$ & $7 \pm 1^{b}(0,93)$ & $18 \pm 1^{\mathrm{a}}(2,25)$ & $14 \pm 1^{a}(1,75)$ \\
\hline 4 & $22 \pm 3^{a}(-)$ & $25 \pm 3^{\mathrm{a}}(-)$ & $34 \pm 3^{a}(-)$ & $34 \pm 3^{a}(-)$ \\
\hline Total (\%) & 85,62 & 85,93 & 66,00 & 67,25 \\
\hline
\end{tabular}

Médias seguidas da mesma letra, não diferem estatisticamente entre si, pelo teste de Tukey $(P>0,01)$; n=número de observações para cada parâmetro. 
TABELA 2 - MÉDIAS E DESVIOS PADRÕES ( \pm ) AJUSTADOS POR QUADRADOS MÍNIMOS PARA O NÚMERO DE ESPERMATOZÓIDES E ÍNDICE DE ATIVIDADE CITOQUÍMICA (IAC), POR CLASSES, DE ACORDO COM HRUDKA (1987), AVALIADAS NO SÊMEN CAPRINO DAS RAÇAS BOER E ALPINA, NO OUTONO E PRIMAVERA, BOTUCATU-SP, 2002.

\begin{tabular}{|c|c|c|c|c|}
\hline \multirow[t]{2}{*}{ Classes } & \multicolumn{2}{|c|}{ Outono } & \multicolumn{2}{|c|}{ Primavera } \\
\hline & $\operatorname{Boer}(\mathrm{n}=41)$ & Alpina $(n=45)$ & Boer $(n=38)$ & Alpina $(n=40)$ \\
\hline 1 & $137 \pm 4^{a}(68,5)$ & $135 \pm 3^{a}(67,5)$ & $141 \pm 5^{\mathrm{a}}(70,5)$ & $140 \pm 4^{\mathrm{a}}(70,0)$ \\
\hline 2 & $18 \pm 2^{\mathrm{a}}(4,5)$ & $23 \pm 1^{a}(5,75)$ & $21 \pm 2^{\mathrm{a}}(5,25)$ & $27 \pm 1^{\mathrm{a}}(6,75)$ \\
\hline 3 & $11 \pm 1^{a}(1,37)$ & $9 \pm 1^{a}(1,12)$ & $10 \pm 10^{a}(1,25)$ & $8 \pm 8^{a}(1,0)$ \\
\hline 4 & $31 \pm 3^{\mathrm{a}}(-)$ & $31 \pm 3^{\mathrm{a}}(-)$ & $26 \pm 4^{a}(-)$ & $23 \pm 3^{a}(-)$ \\
\hline IAC (\%) & 74,37 & 74,37 & 77,00 & 77,75 \\
\hline
\end{tabular}

Médias seguidas da mesma letra, não diferem estatisticamente entre si, pelo teste de Tukey ( $P>0,01)$; n=número de observações para cada parâmetro.

A análise de variância revelou que não houve diferença estatística entre amostras do outono e da primavera (TABELA 1), demonstrando que nas condições do estudo, os machos caprinos das raças Boer e Alpina não apresentaram comportamento reprodutivo estacional. Entretanto, houve efeito significativo entre as amostras pós-colheita e pós-descongelação $(P<0,01)$ nas classes 1, 2 e 3 (TABELA 2).

A distribuição de espermatozóides em várias classes, em diferentes freqüências, demonstra a coexistência de espermatozóides com atividade normal, reduzida e ausente, confirmando a visão geral de que um mesmo ejaculado apresenta um montante de espermatozóides compreendendo células intactas, senescentes e mortas (SALISBURY e VANDENMARK, 1961).

Os índices de atividade citoquímica para amostras pós-colheita, raças Boer e Alpina, foram 85,62 e 85,93 , enquanto que os índices para as amostras pós-descongelação foram 66,00 e 67,25 , para raças Boer e Alpina, respectivamente. Estes índices significam que mais da metade das mitocôndrias estão ativas nos espermatozóides após a descongelação, o que é compatível para suportar o percentual de motilidade espermática quando avaliada após a descongelação. SOOSALU et al. (1975) verificaram ser possível a existência de espermatozóides vivos, mas imóveis.

O índice de atividade citoquímica das amostras pós-descongelação foi numericamente próximo ao verificado por BARBOSA e ESPER (1997) para o sêmen pós-descongelação de touros mestiços, que foi de $65,00 \pm 1,48 \%$.

Conclui-se que os espermatozóides caprinos colhidos em duas estações do ano, e das raças estudadas, apresentam índice de atividade citoquímica similares, o que evidencia um mesmo metabolismo aeróbico dos espermatozóides.

FIGURA 1 - ESPERMATOZÓIDES CAPRINOS CORADOS COM DAB. CATEGORIAS: 1-MITOCÔNDRIAS ATIVAS; 2 - MITOCÔNDRIAS ATIVAS > INATIVAS; 3- MITOCÔNDRIAS ATIVAS < NATIVAS; 4MITOCÔNDRIAS INATIVAS. AUMENTO DE 1000X.

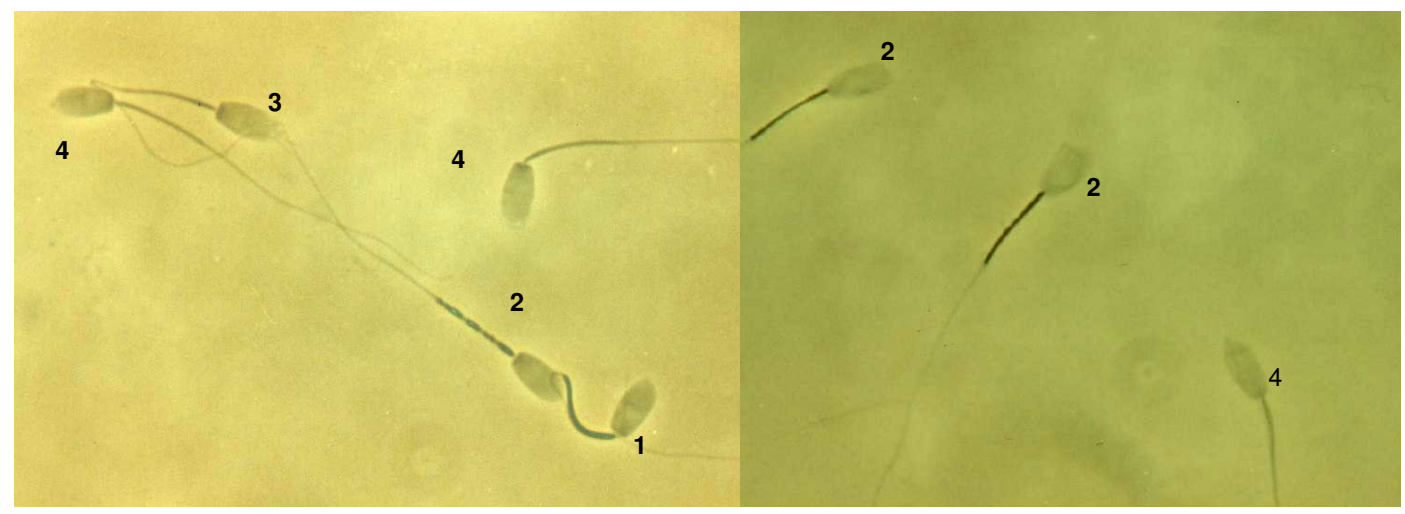


Avaliação da atividade mitocondrial em espermatozóides pós-colheita e pós-descongelação de caprinos...

\section{REFERÊNCIAS}

BARBOSA, R.T.; ESPER, C.R. Avaliação e demonstração da atividade da citocromo $c$ oxidase em espermatozóides bovinos. Ars Veterinaria, Jaboticabal, n. 3, v.13, p.218-223, 1997.

CORREA, J.R.; PACE, M.M., ZAVOS, P.M. Relationships among frozen-thawed sperm characteristics assessed via the routine semen analysis, sperm functional tests and fertility of bulls in artificial insemination program. Theriogenology, New York, v. 48, p. 721-731, 1997.

CUMMINS, J.M.; JEQUIER, A.M.; KAN, R. Molecular biology of the human male infertility: links with aging, mitochondrial genetics and oxidatives stress. Molecular reproduction development, v.37, p.345362, 1994.

EDDY, E.M. The spermatozoa. In: KNOBIL,E.; NEIL, J.D. Physiology of reproduction. New York: raven Press, 1988. p.27-68.

GRAHAM, J. Assessment of sperm quality: a flow cytometric approach. Animal reproduction science, Amsterdam, v.68, p.239-247, 2001.
HRUDKA, F. Cytochemical and ultracytochemical demonstratation of cytochrome c oxidase in spermatozoa and dynamics of its changes accompanying ageing or induced by stress. International Journal of Andrology, v.19, n.6, p.809-828, 1987.

LUKEFAHR, S.D.; HOHENBOKEN, W. Characteristics of spermatozoa midpiece lenght and its relationship with economically important traits incattle. Journal of Dairy Science, Champaign, v.64, p.508-512, 1981.

PEÑA, F.J.; JOHNNISSON, A.; WALLGREN, M.; MARTINEZ RODRIGUEZ. H. Antioxidant supplementation in vitro improves boar sperm motility and mitochondrial membrane potential after cryopreservation of different fractions of the ejaculate. Animal reproduction science, Amsterdam, v.78, p.85-98, 2003.

SALISBURY, G.W.; VANDERMARK, N.L. Physiology of reproduction and artificial insemination of cattle. San Francisco: W.H.Freeman, 1961, $361 \mathrm{p}$.

SOOSALU, O.; EINARSSON, S.; GUSTAFSSON, B. Studies on whole semen and epidymal contents in a bull with low post-thawing sperm motility. Nordisk Veterinaermedicin, Kobenhavn, v.27, p.518-522, 1975.

Recebido para publicação: 30/06/2005

Aprovado:

$20 / 10 / 2005$ 